\title{
ON ANGULAR DERIVATIVE
}

\author{
BY YÛSAKU KoMATU
}

\section{Introduction.}

In order to investigate analytic functions regular in a circle, a representation by means of Poisson-Stieltjes integral due to Herglotz [3] is often useful; cf., for instance, [6], [7], [8], [9]. Let $\Phi(z)$ be an analytic function regular and with positive real part in the unit circle. Then it admits the Herglotz representation

$$
\Phi(z)=\int_{-\pi}^{\pi} \frac{e^{i t}+z}{e^{i t}-z} d \rho(t)+i \Im \Phi(0)
$$

where $\rho(t)$ is a real-valued increasing function defined for $-\pi \leqq t \leqq \pi$. It is further shown that $\rho(t)$ is substantially unique, i. e. it is uniquely determined under the normalization $\rho(-\pi)=0$ and $\rho(t)=(\rho(t-0)+\rho(t+0)) / 2$ for $-\pi<t$ $<\pi$, and it is explicitly given in terms of the referring function by the limit relation

$$
\rho(t)=\lim _{r \rightarrow 1-0} \frac{1}{2 \pi} \int_{-\pi}^{t} \Re \Phi\left(r e^{i \theta}\right) d \theta,
$$

the limit process depending on the continuous parameter $r$; cf. [9]. The total variation of $\rho(t)$ is evidently equal to

$$
\int_{-\pi}^{\pi} d \rho(t)=\Re \Phi(0)
$$

It will be often convenient that $\rho(t)$ is supposed to be continued beyond its original interval of definition by the condition that $\rho(t)-\Re \Phi(0) t / 2 \pi$ has $2 \pi$ as its period. Then the interval of integration may be replaced by any interval with the length $2 \pi$.

Analogue of this representation will plausibly play corresponding roles for studying functions in any other basic domain. For functions harmonic in a half-plane a representation of this sort was really derived by Dinghas [2]. In fact, any function $u(z)$ harmonic and positive in the half-plane $x \equiv \Re z>0$ is representable by the integral formula

$$
u(z)=\frac{x}{\pi} \int_{-\infty}^{\infty} \frac{d \theta(s)}{|z-i s|^{2}}+c x
$$

where $c$ is a non-negative real constant and $\theta(s)$ an increasing function of $s$ which may be, of course, normalized by $\theta(0)=0$. Subsequently, Tsuji [12] gave a simple proof of this representation theorem and further showed that

Received January 29, 1961. 
$\theta(s)$ is unique for every $u(z)$ under the normalization $\theta(s-0)=\theta(s)$. A corresponding result was established also by Verblunsky [14] and Loomis and Widder [11] for the half-plane $y \equiv \Im z>0$.

As shown in these papers [2] and [12], this representation formula offers a simple way to prove the classical Julia-Carathéodory's theorem on the angular derivative. In the form originally obtained by Julia [5] and later refined by Wolff [15], Landau and Valiron [10] etc., it is stated as follows:

Let $f(z)$ be an analytic function regular and with positive real part in the half-plane $\Re z>0$. Then there exists a non-negative real constant $c$ such that it satisfies $\Re f(z) \geqq c \Re z$ for $\Re z>0$, and $f(z) / z \rightarrow c$ and $f^{\prime}(z) \rightarrow c$ uniformly as $z$ tends to $\infty$ through any angular region $|\arg z| \leqq \alpha<\pi / 2$.

The Julia-Carathéodory's theorem just mentioned concerns not only on harmonic but analytic functions. Accordingly, Tsuji [12] completed in the way of his proof the representation for the former to that for the latter. In fact, he used the relation of the complex form

$$
f(z)=\frac{1}{\pi} \int_{-\infty}^{\infty}\left(\frac{1}{z-i s}-\frac{1}{1-i s}\right) d \theta(s)+c z+f(1)-c,
$$

in which the second term of the integrand is inserted in order to ensure the convergence of the infinite integral. Now, corresponding to the Herglotz representation for the case of a circular disc, it seems natural and useful to derive a clearer formula in the complex form valid for the case of a half-plane. The latter will play a role similar to the former which was used by Herzig [4] in discussing the angular derivative of a function regular in a circle.

In the present paper we shall derive a representation formula for analytic functions regular in a half-plane. It is essentially a simple transform of the Herglotz representation but the result obtained will be somewhat neater than the previous Tsuji's one. We shall then try to make some applications of these representation formulas to the angular derivative.

\section{Integral representation.}

We begin with the following representation theorem:

THEOREM 1. Let $f(z)$ be an analytic function regular and with positive real part in the half-plane $\mathfrak{R} z>0$. Then it admits the integral representation

$$
f(z)=\int_{-\infty}^{\infty} \frac{1-i s z}{z-i s} d \lambda(s)+c z+i \Im f(1)
$$

where $c$ is a real constant with $0 \leqq c \leqq \Re f(1)$ and $\lambda(s)$ a real-valued increasing function defined in $-\infty<s<\infty$ and satisfying

$$
\int_{-\infty}^{\infty} d \lambda(s)=\Re f(1)-c .
$$


Proof. By means of the linear transformation $z=(1+Z) /(1-Z)$, i. e. $Z=(z-1) /(z+1)$, the function $f(z)$ becomes an analytic function

$$
\Phi(Z)=f\left(\frac{1+Z}{1-Z}\right)
$$

regular and with positive real part in the unit circle $|Z|<1$. Hence it is represented by means of the Herglotz formula

$$
\Phi(Z)=\int_{0}^{2 \pi} \frac{e^{i t}+Z}{e^{i t}-Z} d \rho(t)+i \Im \Phi(0)
$$

where $\rho(t)$ is a real-valued increasing function defined for $0 \leqq t \leqq 2 \pi$ and with the total variation equal to $\Re \Phi(0)$. Substituting $Z=(z-1) /(z+1)$ and accordingly changing the integration variable by means of

$$
i s=\frac{1+e^{i t}}{1-e^{i t}}, \quad \text { i. e. } \quad s=\cot \frac{t}{2},
$$

it readily becomes the desired formula in which $\lambda(s)$ is a real-valued increasing function defined by

$$
\lambda(s)=\rho(2 \pi-0)-\rho(2 \operatorname{arccot} s)
$$

with $\pi>\operatorname{arccot} s>0$. The constant $c$ is evidently given by

$$
c=\rho(+0)-\rho(0)+\rho(2 \pi)-\rho(2 \pi-0),
$$

or $c=\rho(+0)-\rho(-0)$ in view of the periodic continuation of $\rho(t)-\Re \Phi(0) t / 2 \pi$. Finally, we have the relation

$$
\Re f(1)=\int_{-\infty}^{\infty} d \lambda(s)+c
$$

which implies, in paticular, $0 \leqq c \leqq \Re f(1)$.

By the way, it is remarked that the formula used by Tsuji may be written in the form

$$
f(z)=\frac{1}{\pi} \int_{-\infty}^{\infty}\left(\frac{1-i s z}{z-i s}-1\right) \frac{d \theta(s)}{1+s^{2}}+c z+f(1)-c .
$$

Hence, by putting

$$
\frac{1}{\pi} \frac{d \theta(s)}{1+s^{2}}=d \lambda(s)
$$

it is further transformed into

$$
f(z)=\int_{-\infty}^{\infty} \frac{1-i s z}{z-i s} d \lambda(s)+c z+f(1)-c-\int_{-\infty}^{\infty} d \lambda(s)
$$

The formula established in theorem 1 shows moreover that the additional constant terms in the right-hand member must actually reduce to $i \Im f(1)$.

The uniqueness assertion of $\lambda(s)$ is an immediate consequence from that of $\rho(t)$; cf. [9]. It may be stated as follows: 
THEOREM 2. The function $\lambda(s)$ involved in the representation formula of theorem 1 is substantially unique. Namely, it is uniquely determined for every $f(z)$ under consideration, provided it is normalized by

$$
\lambda(s) \rightarrow 0 \text { as } s \rightarrow-\infty \text { and } \lambda(s)=\frac{1}{2}(\lambda(s-0)+\lambda(s+0)) \text { for }-\infty<s<\infty .
$$

Moreover, it is explicitly expressed by the limit relation

$$
\lambda(s)=\lim _{r \rightarrow 1-0} \frac{1}{2 \pi} \int_{2 \operatorname{arccot} s}^{2 \pi} \Re f\left(\frac{1+r e^{i \theta}}{1-r e^{i \theta}}\right) d \theta
$$

with $\pi>\operatorname{arccot} s>0$.

\section{Fundamental theorems on angular derivative.}

Now, the representation formula for $f(z)$ having been established, the Julia-Carathéodory's theorem in the form stated by Landau and Valiron will be readily derived. While this theorem concerns a function itself and its derivative of the first order, analogous behavior of derivative of any higher order can be also obtained. Thus we shall give here a proof of the theorem which is supplemented by the behaviors of derivatives of higher order. The original part was proved also by Tsuji [12] in a similar way.

THEOREM 3. Let $f(z)$ be an analytic function regular and with positive real part in the half-plane $\Re z>0$. Then there exists a non-negative real constant $c$ for which we have $\Re f(z) \geqq c \Re z$ for $\Re z>0$, or more precisely

$$
c=\inf _{\Re z>0} \frac{\Re f(z)}{\Re z}=\inf _{x>0} \frac{\Re f(x)}{x} .
$$

The equality $\Re f(z)=c \Re z$ holds at a point if and only if $f(z)=c z+i \Im f(1)$. Moreover, for the constant $c$ thus determined, the function $f(z)$ and its successive derivatives satisfy the limit relations

$$
z^{n-1} f^{(n)}(z) \rightarrow \begin{cases}c & (n=0,1), \\ 0 & (n=2,3, \cdots)\end{cases}
$$

valid uniformly as $z$ tends to $\infty$ through any angular region $|\arg z| \leqq \alpha$ $<\pi / 2$.

Proof. The representation formula in theorem 1 yields, after separating the real part,

$$
\Re f(z)=\Re z \cdot \int_{-\infty}^{\infty} \frac{1+s^{2}}{|z-i s|^{2}} d \lambda(s)+c \Re z .
$$

The first half of the theorem is an immediate consequence of the last relation; in particular, if the equality $\Re f(z)=c \Re z$ holds at a point, $\lambda(s)$ must remain constant and hence $f(z)=c z+i \Im f(1)$.

Now, since $|z-i s| \geqq|s| \cos \alpha$ holds for $|\arg z| \leqq \alpha<\pi / 2$, the formula in theorem 1 yields the estimation 


$$
\left|\frac{f(z)}{z}-c\right| \leqq \frac{1}{|z|} \int_{\mid s ; \leq s}\left|\frac{1-i s z}{z-i s}\right| d \lambda(s)+2 \sec \alpha \cdot \int_{\mid s,>s} d \lambda(s)+\frac{|\Im f(1)|}{|z|},
$$

provided $S|z| \geqq 1$. Hence, taking $S$ large enough and then letting $z$ tend to $\infty$, we see that $f(z) / z$ tends to $c$ uniformly. Similarly, from the derived formula

$$
f^{\prime}(z)=-\int_{-\infty}^{\infty} \frac{1+s^{2}}{(z-i s)^{2}} d \lambda(s)+c
$$

we get the estimation

$$
\left|f^{\prime}(z)-c\right| \leqq \int_{\mid s ; \leqq s} \frac{1+s^{2}}{|z-i s|^{2}} d \lambda(s)+2 \sec ^{2} \alpha \cdot \int_{|s|>s} d \lambda(s),
$$

provided $S \geqq 1$. It follows that $f^{\prime}(z)$ tends to $c$ uniformly.

For any integer $n \geqq 2$, we have

and hence

$$
f^{(n)}(z)=(-1)^{n} n ! \int_{-\infty}^{\infty} \frac{1+s^{2}}{(z-i s)^{n+1}} d \lambda(s)
$$

$$
z^{n-1} f^{(n)}(z)=(-1)^{n} n ! \int_{-\infty}^{\infty} \frac{1+s^{2}}{(z-i s)^{2}} \sum_{\nu=0}^{n-1}\left(\begin{array}{c}
n-1 \\
\nu
\end{array}\right)\left(\frac{i s}{z-i s}\right)^{\nu} d \lambda(s)
$$

Consequently, we get similarly as above the estimation

$$
\begin{aligned}
& \left|z^{n-1} f^{(n)}(z)\right| \\
\leqq & n !\left(\int_{|s| \leq s} \frac{|z|^{n-1}\left(1+s^{2}\right)}{|z-i s|^{n+1}} d \lambda(s)+\int_{|s|>S} \frac{1+s^{2}}{s^{2} \cos ^{2} \alpha} \sum_{\nu=0}^{n-1}\left(\begin{array}{c}
n-1 \\
\nu
\end{array}\right) \frac{1}{\cos ^{\nu} \alpha} d \lambda(s)\right) \\
\leqq & n !\left(\int_{|s| \leq s} \frac{|z|^{n-1}\left(1+s^{2}\right)}{|z-i s|^{n+1}} d \lambda(s)+2 \sec ^{2} \alpha(1+\sec \alpha)^{n-1} \int_{|s|>S} d \lambda(s)\right),
\end{aligned}
$$

provided $S \geqq 1$. This shows, based on the argument employed above, that $z^{n-1} f^{(n)}(z)$ tends to 0 uniformly as $z \rightarrow \infty$ along any Stolz path through the angular region.

It is noted that the branch of $f(z)=z^{m}$ with $0<m<1$ determined by $f(1)=1$ satisfies the condition imposed in theorem 3 and its derivative $f^{(n)}(z)$ is equal to a non-zero constant multiple of $z^{m-n}$. Since $m$ may be taken arbitrarily near the unity, the power $n-1$ of $z$ in the expression $z^{n-1} f^{(n)}(z)$ cannot be ameliorated.

In case of a circular disc as the basic domain, the fundamental theorem takes the form mentioned by Carathéodory [1]. For deriving this, it will be in principle only necessary to transform theorem 3 by means of linear functions. But in transferring the proposition on the derivatives of higher order, the actual computation together with necessary estimations will be perhaps somewhat troublesome. Accordingly, by making use of the Herglotz representation, we shall give here a direct proof of the theorem which is supplemented by the behaviors of derivatives of higher order. Though the original part mentioned by Carathéodory was already proved also by Herzig [4] in a similar 
way, we reproduce it here briefly for the sake of completeness.

THEOREM 4. Let $F(z)$ be an analytic function regular and satisfying $|F(z)|<1$ in the unit circle $|z|<1$. Then there exists a positive real constant $D$, eventually equal to $+\infty$, i.e. the so-called angular derivative of $F(z)$ at $z=1$, for which we have

$$
\frac{1-|F(z)|^{2}}{|1-F(z)|^{2}} \geqq \frac{1}{D} \frac{1-|z|^{2}}{|1-z|^{2}} \quad \text { for } \quad|z|<1,
$$

$D$ being understood as the least possible constant satisfying this inequality. Here the equality holds at a point if and only if $F(z)$ is a linear function defined by

$$
\frac{1+F(z)}{1-F(z)}=\frac{1}{D} \frac{1+z}{1-z}+i \beta,
$$

$\beta=\Im((1+F(0)) /(1-F(0)))$ being a real constant; in this case, $D$ is necessarily finite. In general, the function satisfies the limit relation

$$
\frac{1-F(z)}{1-z} \rightarrow D
$$

and, if $D$ is supposed finite, its successive derivatives satisfy the limit relations

$$
F^{\prime}(z) \rightarrow D \quad \text { and } \quad(1-z)^{n-1} F^{(n)}(z) \rightarrow 0 \quad(n=2,3, \cdots),
$$

every relation holding uniformly as $z$ tends to 1 along any path through a Stolz angle in $|z|<1$ with the vertex at $z=1$.

Proof. Since $(1+F(z)) /(1-F(z))$ is regular and with positive real part in $|z|<1$, the Herglotz representation yields

$$
\frac{1+F(z)}{1-F(z)}=\int_{0}^{2 \pi} \frac{e^{i t}+z}{e^{i t}-z} d \rho(t)+i \Im \frac{1+F(0)}{1-F(0)},
$$

$\rho(t)$ being a real-valued increasing function defined for $0 \leqq t \leqq 2 \pi$ with the total variation equal to $\Re((1+F(0)) /(1-F(0)))$. By separating the real part, we get

$$
\begin{aligned}
& \frac{1-|F(z)|^{2}}{\mid 1-\overline{\left.F(z)\right|^{2}}}=\int_{0}^{2 \pi} \frac{1-|z|^{2}}{\left|e^{i t}-z\right|^{2}} d \rho(t) \\
\geqq & \left(\int_{0}^{+0}+\int_{2 \pi-0}^{2 \pi}\right) \frac{1-|z|^{2}}{\left|e^{i t}-z\right|^{2}} d \rho(t)=\frac{1}{D} \frac{1-|z|^{2}}{|1-z|^{2}}
\end{aligned}
$$

where $D$ is given by

$$
\frac{1}{D}=\rho(+0)-\rho(0)+\rho(2 \pi)-\rho(2 \pi-0)=\rho(+0)-\rho(-0)
$$

in view of the continuation of $\rho(t)$ mentioned before. In particular, we have

$$
0 \leqq \frac{1}{D} \leqq \int_{0}^{2 \pi} d \rho(t)=\Re \frac{1+F(0)}{1-F(0)}<+\infty \text {, i. e. } 0<D \leqq+\infty .
$$


The equality in the above inequality holds if and only if $\rho(t)$ remains constant in the interval $0<t<2 \pi$. Hence the extremal function must be a linear iunction given in the theorem.

Next, for any $F(z)$ under consideration, it holds

$$
\begin{aligned}
\frac{1}{1-F(z)} & =\int_{0}^{2 \pi} \frac{e^{i t}}{e^{i t}-z} d \rho(t)-\frac{F(0)}{1-\bar{F}(0)} \\
& =\frac{1}{D} \frac{1}{1-z}+\int_{+0}^{2 \pi-0} \frac{e^{2 t}}{e^{2 t}-z} d \rho(t)-\frac{F(0)}{1-F(0)} .
\end{aligned}
$$

For $z$ tending to 1 along a Stolz path, an estimation of the form $|1-z| /(1$ $-|z|) \leqq K<+\infty$ holds. Therefore, for any $\delta$ with $0<\delta<\pi$, we obtain

$$
\begin{aligned}
& \left|\frac{1-z}{1-F(z)}-\frac{1}{D}\right| \\
\leqq & K\left(\int_{+0}^{\delta}+\int_{2 \pi-\delta}^{2 \pi-0}\right) d i(t)+|1-z|\left(\int_{\delta}^{2 \pi-\delta} \frac{1}{\left|e^{2 t}-z\right|} d \rho(t)+\left|\frac{F(0)}{1-F(0)}\right|\right) .
\end{aligned}
$$

Consequently, taking $\delta$ small enough and then letting $z$ tend to 1 , it follows that the limit relation $(1-z) /(1-F(z)) \rightarrow 1 / D$, i. e. $(1-F(z)) /(1-z) \rightarrow D$ holds uniformly. In quite a similar way, it follows from the derived formula

$$
\frac{F^{\prime}(z)}{(1-F(z))^{2}}=\int_{0}^{2 \pi} \frac{e^{i t}}{\left(e^{i t}-z\right)^{2}} d \rho(t)
$$

that the limit relation

$$
\left(\frac{1-z}{1-F(z)}\right)^{2} F^{\prime}(z) \rightarrow \frac{1}{D}
$$

holds uniformly. Hence, by combining with the relation established above, we obtain the relation $F^{\prime}(z) \rightarrow D$ provided $D<+\infty$, valid uniformly.

Finally, suppose $n \geqq 2$ and $D<+\infty$. The Herglotz representation for (1 $+F(z)) /(1-F(z))$ or equivalently for $1 /(1-F(z))$ becomes after differentiating $n$ time

$$
\begin{aligned}
\frac{d^{n}}{d z^{n}} \frac{1}{1-F(z)} & =n ! \int_{0}^{2 \pi} \frac{e^{i t}}{\left(e^{i t}-z\right)^{n+1}} d i(t) \\
& =\frac{n !}{D} \frac{1}{(1-z)^{n+1}}+n ! \int_{+0}^{2 \pi-0} \frac{e^{i t}}{\left(e^{i t}-z\right)^{n+1}} d \rho(t) .
\end{aligned}
$$

Hence we conclude by means of a similar argument as above that the limit relation

$$
(1-z)^{n+1} \frac{d^{n}}{d z^{n}} \frac{1}{1-F(z)} \rightarrow \frac{n !}{D}
$$

holds uniformly for Stolz approach. Now, it is readily verified by induction that the $n$th derivative of $1 /(1-F(z))$ with respect to $z$ is a polynomial of $n$ arguments $\omega_{\nu} \equiv F^{(\nu)}(z) /(1-F(z)) \quad(\nu=1, \cdots, n)$ multiplied by $1 /(1-F(z))$, the coefficients of the polynomial being numerical constants independent of $F(z)$. Further, if $\omega_{\nu}$ is regarded as a quantity of degree $\nu$ respectively, then this 
polynomial is homogeneous with the degree $n$. In other words, the $n$th derivative in question is of the form

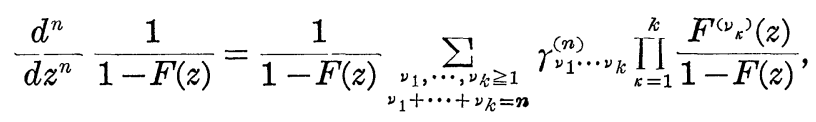

the $r$ 's being numerical constants independent of $F(z)$. Evidently, we obtain, in particular,

$$
\gamma_{n}^{(n)}=1 \quad \text { and } \quad \gamma_{1 \cdots 1}^{(n)}=n !
$$

In order to prove the last part of the theorem by induction, we first deal with the case $n=2$ directly. For this case we have

$$
\frac{d^{2}}{d z^{2}} \frac{1}{1-F(z)}=\frac{F^{\prime \prime}(z)}{(1-F(z))^{2}}+\frac{2 F^{\prime}(z)^{2}}{(1-F(z))^{3}} \text {. }
$$

Consequently, in view of the limit relation established above for general $n \geqq 2$, it follows that

$$
\begin{aligned}
\frac{2}{D^{2}} & =\lim _{z \rightarrow 1}(1-z)^{3} \frac{d^{2}}{d z^{2}} \frac{1}{1-F(z)} \\
& =\lim _{z \rightarrow 1}\left(\left(\frac{1-z}{1-F(z)}\right)^{2}(1-z) F^{\prime \prime}(z)+2\left(\frac{1-z}{1-F(z)}\right)^{3} F^{\prime}(z)^{2}\right) \\
& =\frac{1}{D^{2}} \lim _{z \rightarrow 1}(1-z) F^{\prime \prime}(z)+2 \frac{1}{D^{3}} \cdot D^{2},
\end{aligned}
$$

i. e.

$$
\lim _{z \rightarrow 1}(1-z) F^{\prime \prime}(z)=0
$$

holds uniformly for Stolz approach. Suppose now

$$
\lim _{z \rightarrow 1}(1-z)^{\nu-1} F^{(\nu)}(z)=0 \quad \text { for } \quad \nu=2, \cdots, n-1
$$

uniformly for Stolz approach. Then, we obtain

$$
\begin{aligned}
\frac{n !}{D} & =\lim _{z \rightarrow 1}(1-z)^{n+1} \frac{d^{n}}{d z^{n}} \frac{1}{1-F(z)} \\
& =\lim _{z \rightarrow 1}\left(\frac{1-z}{1-F(z)} \sum_{\substack{\nu_{1}, \cdots, \nu_{k} \geq 1 \\
\nu_{1}+\cdots+\nu_{k}=n}} \gamma_{\nu_{1} \cdots \nu_{k}}^{(n)} \prod_{\kappa=1}^{k} \frac{1-z}{1-F(z)}(1-z)^{\nu_{k}-1} F^{\left(\nu_{k}\right)}(z)\right) \\
& =\frac{1}{D} \lim _{z \rightarrow 1}\left(\frac{1}{D}(1-z)^{n-1} F^{(n)}(z)+n ! \frac{1}{D^{n}}-F^{\prime}(z)^{n}\right) \\
& =\frac{1}{D^{2}} \lim _{z \rightarrow 1}(1-z)^{n-1} F^{(n)}(z)+\frac{n !}{D} .
\end{aligned}
$$

This shows that the desired relation $(1-z)^{n-1} F^{(n)}(z) \rightarrow 0$ holds uniformly in general for $n \geqq 2$ as $z$ tends to 1 along any Stolz path.

It is noted that the branch of $F(z)=1-4 /\left(\zeta+\zeta^{m}+2\right)$ with $\zeta \equiv(1+z) /(1$ $-z$ ) and $0<m<1$ determined by $F(0)=0$ satisfies the condition imposed in theorem 4. Its angular derivative at $z=1$ has a finite value $D=2$. On the 
other hand, it is verified that its $n$th derivative $F^{(n)}(z)$ grows near $z=1$ with the order of $(1-z)^{-n+2-m}$. Since $m$ can be taken arbitrarily near the unity, the exponent of $1-z$ in the expression $(1-z)^{n-1} F^{(n)}(z)$ cannot be ameliorated.

\section{Estimation of angular derivative.}

In theorem 4, if $F(z)$ is subject to the condition $F(0)=0$, it follows that its angular derivative $D$ at $z=1$ satisfies $D \geqq 1$ and the equality here holds if and only if $F(z) \equiv z$. Under an additional condition that $F^{\prime}(0)$ has a preassigned value, a more precise estimation was derived by Unkelbach [13] and soon later re-proved by Herzig [4]. Since by Schwarz's lemma we have always $\left|F^{\prime}(0)\right| \leqq 1$ with the equality valid for $F(z) \equiv F^{\prime}(0) z$ alone, the case $\left|F^{\prime}(0)\right|<1$ only is essential. The result of Unkelbach states that

$$
D \geqq 2 \frac{1-\Re F^{\prime}(0)}{1-\left|F^{\prime}(0)\right|^{2}} \quad(>1)
$$

and that the equality holds if and only if

$$
F(z)=z \frac{\left(1-F^{\prime}(0)\right) z+F^{\prime}(0)\left(1-\overline{F^{\prime}(0)}\right)}{F^{\prime}(0)\left(1-F^{\prime}(0)\right) z+1-\bar{F}^{\prime}(0)} .
$$

Now, we shall show that this result can be generalized as in the following manner.

THEOREM 5. Let $F(z)$ be an analytic function regular and satisfying $|F(z)|<1$ in $|z|<1$. Then we have an inequality

$$
\left|\frac{F^{\prime}(z)}{(1-F(z))^{2}}-\frac{1}{D} \frac{1}{(1-z)^{2}}\right| \leqq\left(\Re \frac{1+F(0)}{1-F(0)}-\frac{1}{D}\right) \frac{1}{(1-|z|)^{2}}
$$

valid for any $z$ in $|z|<1$. At any assigned $z_{0}=\left|z_{0}\right| e^{i \theta_{0}}$ in $|z|<1$, the equality holds if and only if $F(z)$ is a rational function satisfying

$$
\frac{1}{1-F(z)}=\frac{1}{D} \frac{1}{1-z}+\left(\Re \frac{1+F(0)}{1-F(0)}-\frac{1}{D}\right) \frac{e^{i \theta_{0}}}{e^{i \theta} \theta_{0}-z}-\frac{F(0)}{1-F(0)} .
$$

When the value of $F^{\prime}\left(z_{0}\right) /\left(1-F_{0}(z)\right)^{2}$ is preassigned, the value of $D$ for the extremal function may be determined by the equation

$$
\frac{F^{\prime}\left(z_{0}\right)}{\left(1-F\left(z_{0}\right)\right)^{2}}=\frac{1}{D} \frac{1}{\left(1-z_{0}\right)^{2}}+\left(\Re \frac{1+F(0)}{1-F(0)}-\frac{1}{D}\right) \frac{e^{i \theta_{0}}}{\left(e^{2 \theta_{0}}-z_{0}\right)^{2}} .
$$

Proof. From a relation used in the proof of theorem 4 we get

$$
\frac{F^{\prime}(z)}{(1-F(z))^{2}}=\frac{1}{D} \frac{1}{(1-z)^{2}}+\int_{+0}^{2 \pi-0} \frac{e^{i t}}{\left(e^{i t}-z\right)^{2}} d \rho(t) .
$$

Thus, evident relations

$$
\left|\frac{e^{i t}}{\left(e^{i t}-z\right)^{2}}\right| \leqq \frac{1}{(1-|z|)^{2}}, \quad d \rho(t) \geqq 0, \quad \int_{+0}^{2 \pi-0} d_{i} \rho(t)=\Re \frac{1+F(0)}{1-F(0)}-\frac{1}{D}
$$

imply immediately the desired results. 
Unkelbach's estimation of $D$ is regarded as a particular case of the theorem. We have only to put $F(0)=0$ and $z=0$. In fact, we then obtain

$$
\left|F^{\prime}(0)-\frac{1}{D}\right| \leqq 1-\frac{1}{D}, \quad \text { i. e. } \quad D \geqq 2 \frac{1-\Re F^{\prime}(0)}{1-\left|F^{\prime}(0)\right|^{2}} .
$$

For preassigned value of $F^{\prime}(0)$, the extremal function is defined by

$$
\frac{1}{1-F(z)}=\frac{1}{D} \frac{1}{1-z}+\left(1-\frac{1}{D}\right) \frac{e^{i \theta_{0}}}{e^{i \theta_{0}}-z}
$$

with $F^{\prime}(0)=1 / D+(1-1 / D) e^{-i \theta_{0}}$. Hence, inserting the values

$$
\frac{1}{D}=\frac{1-F^{\prime}(0) \overline{F^{\prime}(0)}}{2-F^{\prime}(0)-\bar{F}^{\prime}(0)} \quad \text { and } \quad e^{\imath \theta_{0}}=\frac{\overline{F^{\prime}(\overline{0})}-1 / D}{1-1 / D}=-\frac{1-\bar{F}^{\prime}(0)}{1-F^{\prime}(0)},
$$

we really obtain

$$
F(z)=z^{\left(1-F^{\prime}(0)\right) z+F^{\prime}(0)\left(1-\bar{F}^{\prime}(0)\right)}
$$

In a similar way, we can derive an inequality involving higher derivatives.

THEOREM 6. For $F(z)$ considered in theorem 5, we have, for any integer $n \geqq 1$, an inequality

$$
\left|\frac{1}{n !} \frac{d^{n}}{d z^{n}} \frac{1}{1-F(z)}-\frac{1}{D} \frac{1}{(1-z)^{n+1}}\right| \leqq\left(\Re \frac{1+F(0)}{1-F(0)}-\frac{1}{D}\right) \frac{1}{(1-|z|)^{n+1}} .
$$

At any assigned $z_{0}=\left|z_{0}\right| e^{i \theta_{0}}$ in $|z|<1$, the equality holds if and only if $F(z)$ satisfies

$$
\frac{1}{1-F(z)}=\frac{1}{D} \frac{1}{1-z}+\left(\Re \frac{1+F(0)}{1-F(0)}-\frac{1}{D}\right) \frac{e^{i \theta_{0}}}{e^{i \theta_{0}-z}}-\frac{\overline{F(0)}}{1-\overline{F(0)}}
$$

when $z_{0} \neq 0$ while it satisfies

$$
\frac{1}{1-F(z)}=\frac{1}{D} \frac{1}{1-z}+\sum_{j=0}^{n-1} \rho_{j} \frac{e^{2\left(t_{0}+2 j \pi / n\right)}}{e^{i\left(t_{0}+2 j \pi / n\right)}-z}-\frac{\bar{F}(0)}{1-\overline{F(0)}}
$$

when $z_{0}=0$ where $t_{0}$ is any real number and the $\rho$ 's denote arbitrary real numbers subject to the conditions

$$
\rho_{j} \geqq 0(j=0, \cdots, n-1) \quad \text { and } \quad \sum_{j=0}^{n-1} \rho_{j}=\Re \frac{1+F(0)}{1-F(0)}-\frac{1}{D} .
$$

Proof. We can proceed similarly as for theorem 5. In fact, we have only to remember

$$
\frac{1}{n !} \frac{d^{n}}{d z^{n}} \frac{1}{1-F(z)}=\frac{1}{D} \frac{1}{(1-z)^{n+1}}+\int_{+0}^{2 \pi-0} \frac{e^{i t}}{\left(e^{i t}-z\right)^{n+1}} d \rho(t) .
$$

It would be remarked here by the way that, by means of the notations used in the proof of theorem 4, we have 


$$
\left[\frac{d^{n}}{d z^{n}} \frac{1}{1-F(z)}\right]^{z=0}=\frac{1}{1-F(0)} \sum_{\substack{\nu_{1}, \cdots, \nu_{k} \geq 1 \\ \nu_{1}+\cdots+\nu_{k}=n}} \gamma_{\nu_{1} \cdots \nu_{k}}^{(n)} \prod_{k=1}^{k} \frac{F^{\left(\nu_{\kappa}\right)}(0)}{1-F(0)} \text {. }
$$

On the other hand, if $F(z)$ vanishes at the origin, we may replace $1 /(1-F(z))$ in the left-hand member by $\sum_{\nu=0}^{n} F(z)^{\nu}$. Thus, in this case, we obtain an alternative relation generating the $r$ 's:

$$
\sum_{\nu=1}^{n}\left[\frac{d^{n}}{d z^{n}} F(z)^{\nu}\right]^{z=0}=\sum_{\substack{\nu_{1}, \cdots, \nu_{k} \geq 1 \\ \nu_{1}+\cdots+\nu_{k}=n}} \gamma_{\nu_{1} \cdots \nu_{k}}^{(n)} \prod_{\kappa=1}^{k} F^{\left(\nu_{k}\right)}(0) .
$$

Since the last identity remains valid for any analytic function $F(z)$ vanishing at the origin, it will serve the actual determination of the values of the r's.

Corresponding to theorem 5 or 6 , similar and essentially equivalent inequalities are derived more readily for the case of a half-plane. For the sake of brevity, we restrict ourselves to formulate an estimation corresponding to Unkelbach's.

THEOREM 7. Let $f(z)$ be an analytic function regular and with positive real part in $\Re z>0$. Then we have an estimation

$$
c \leqq \frac{1}{2} \frac{(\Re f(1))^{2}-\left|f^{\prime}(1)\right|^{2}}{\Re f(1)-\Re f^{\prime}(1)}
$$

provided $f(z) \neq c z+i \Im f(1)$. The equality holds here if and only if $f(z)$ is a rational function of the form

$$
f(z)=(\Re f(1)-c) \frac{1-i s_{0} z}{z-i s_{0}}+c z+i \Im ⿱ 乛 ⿻ 上 丨(1),
$$

$s_{0}$ being any real constant. In particular, if $f(z)$ is subject to the condition $f(1)=1$ and the value of $f^{\prime}(1)$ necessarily satisfying $\left|f^{\prime}(1)\right|<1$ is preassigned, the estimation becomes

$$
c \leqq \frac{1}{2} \frac{1-\left|f^{\prime}(1)\right|^{2}}{1-\Re f^{\prime}(1)} \quad(<1)
$$

and the extremal function is of the form

$$
f(z)=z-\frac{\left|1-f^{\prime}(1)\right|^{2}}{2} \frac{z^{2}-1}{\left(1-\Re f^{\prime}(1)\right) z+i \Im f^{\prime}(1)} .
$$

Proof. The representation formula established in theorem 1 yields, after differentiation with respect to $z$ followed by putting $z=1$, the relation

$$
f^{\prime}(1)=-\int_{-\infty}^{\infty} \frac{1+i s}{1-i s} d \lambda(s)+c
$$

Hence it follows readily that

$$
\left|f^{\prime}(1)-c\right| \leqq \int_{-\infty}^{\infty} d \lambda(s)=\Re f(1)-c .
$$

Since this implies, in particular, the inequality $\Re f^{\prime}(1) \leqq\left|f^{\prime}(1)\right| \leqq \Re f(1)$ with 
the last equality only for $f(z)=c z+i \Im f(1)$, we obtain the desired estimation. Extremal function appears if and only if $\lambda(s)$ jumps at a single point, $s_{0}$ say, and hence it is of the form stated in the theorem. If $f(z)$ satisfies $f(1)=1$ and the value of $f^{\prime}(1)$ is preassigned, then the values of $c$ and $s_{0}$ for the extremal function are determined by the equations

$$
c=\frac{1}{2} \frac{1-\left|f^{\prime}(1)\right|^{2}}{1-\Re f^{\prime}(1)} \text { and } f^{\prime}(1)=-\frac{1+i s_{0}}{1-i s_{0}}(1-c)+c .
$$

Inserting the values thus determined, we obtain the last mentioned form of $f(z)$.

In a similar way, we can derive for any $n \geqq 2$ an analogous estimation involving $f^{(n)}(1)$. For instance, if $f(z)$ satisfies the condition at the beginning of theorem 7 together with $\Re f(1)=1$, we obtain

$$
\left|f^{(n)}(1)\right|=\left|(-1)^{n} n ! \int_{-\infty}^{\infty} \frac{1+i s}{(1-i s)^{n}} d \lambda(s)\right| \leqq n !(1-c) ;
$$

here the equality holds if and only if $f(z)$ is a rational function of the form

$$
f(z)=\frac{1-c}{z}+c z+i \Im f(1)
$$

\section{REFERENCES}

[1] Carathéodory, C., Über die Winkelderivierten von beschränkten analytischen Funktionen. Sitzungsber. Preuss. Akad. Wiss. (1929), 1-18.

[2] Dinghas, A., Über das Phragmén-Lindelöfsche Prinzip und den Julia-Carathéodoryschen Satz. Sitzungsber. Preuss. Akad. Wiss. (1938), 32-48.

[3] Herglotz, A., Über Potenzreihen mit positivem, reellem Teil im Einheitskreise. Leipziger Ber. 63 (1911), 501-511.

[4] Herzig, A., Die Winkelderivierte und das Poisson-Stieltjes-Integral. Math. Zeitschr. 46 (1940), 129-156.

[5] Julia, G., Extensions nouvelles d'un lemme de Schwarz. Acta Math. 42 (1920), 349-355.

[6] Komatu, Y., Einige Darstellungen analytischer Funktionen und ihre Anwendungen auf konforme Abbildung. Proc. Imp. Acad. Tokyo 20 (1944), 536-541.

[7] Komatu, Y., On analytic functions with positive real part in a circle. Kōdai Math. Sem. Rep. 10 (1958), 64-83.

[8] Komatu, Y., On the range of analytic functions with positive real part. Kōdai Math. Sem. Rep. 10 (1958), 145-160.

[9] Komatu, Y., On the integral representation of Herglotz and the trigonometric moment problem. Jap. Journ. Math. 29 (1959), 35-42.

[10] Landau, E., AND G. Valiron, A deduction from Schwarz's lemma. Journ. London Math. Soc. (4) 4 (1929), 162-163.

[11] Loomis, H. L., AND D. V. WIDDER, The Poisson integral representation of functions which are positive and harmonic in a half-plane. Duke Math. Journ. 9 (1942), 643-645. 
[12] TsuJI, M., On a positive harmonic function in a half-plane. Jap. Journ. Math. 15 (1939), 277-285.

[13] UNKelbaCh, H., Über die Randverzerrung bei! konformer Abbildung. Math. Zeitschr. 43 (1938), 739-742.

[14] Verblunsky, S., On positive harmonic functions in a half-plane. Proc. Cambridge Phil. Soc. 31 (1935), 482-507.

[15] WolfF, J., Sur une généralisation d'un théorème de Schwarz. C. R. Paris 183 (1926), 500-502.

Department of Mathematics,

Tokyo Institute of TeChNology. 